\title{
The building blocks of matter
}

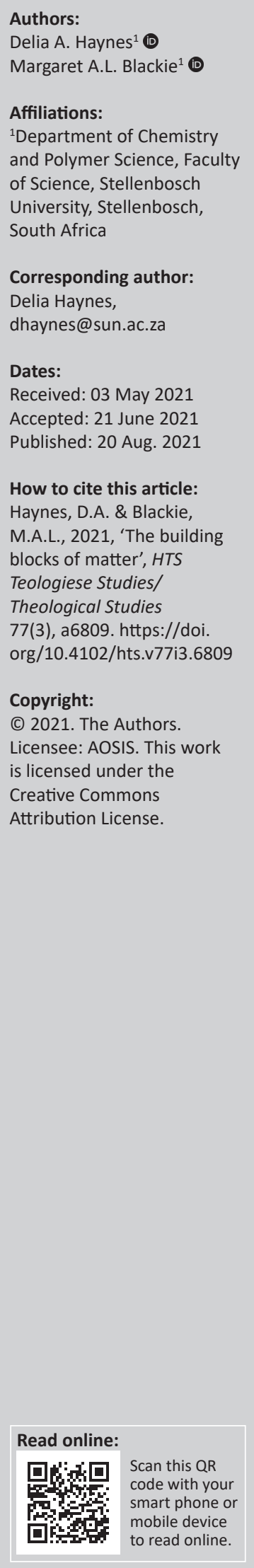

The question of what everything around us is made from has fascinated humanity since ancient times. The development of ideas on what the building blocks of matter are, and how these building blocks come together to form materials, are discussed in this contribution. In order to manipulate matter in a useful way, an understanding of the structure of the building blocks is the key. For this reason, atomic theory and bonding theory are introduced. An explanation of the periodic table shows why it is such a powerful predictive tool, and the use of symbols in chemistry is discussed.

Contribution: In this article, the historical development of key ideas in our understanding of matter is presented, along with some of the important ideas in understanding how matter behaves. As part of this special collection, the implications of the meaning and utilisation of this knowledge are also considered.

Keywords: matter; chemistry; atomic structure; bonding; materials; quantum mechanics; states of matter; symbols.

\section{Introduction}

The Universe, as far as we know, is made from only a few fundamental constituents: dark matter, dark energy, radiation and ordinary matter. Here, we are concerned with ordinary matter, which makes up a tiny proportion of the Universe (see the article by A. J. John [2021] in this special collection for more about the rest of the Universe). Ordinary matter is the stuff of which everything around us is made - tables, potatoes, clouds, planets and people - and this matter is the same throughout the Universe. The stars and planets are made from the same basic building blocks as you and I. Matter is defined as anything that has mass and occupies space (OED Online 2021). The study of matter, its behaviour and the changes it undergoes is known as chemistry. Any investigation of the building blocks of matter will thus involve chemistry.

Every day in the process of simply going about living life, we encounter chemistry. From the burning of fuel in an internal combustion engine which so many rely on for transport, to cooking a meal, many common processes involve chemical reactions. The simple act of boiling a kettle involves liquid water molecules being heated until they reach the boiling point and move into the gas phase. Despite being surrounded by chemistry, most people have no chemical 'intuition' (Blackie 2014:462-469). We simply do not see molecular interactions. And yet every breath we take is an exchange of small molecules: carbon dioxide, the product of chemical reactions occurring in our cells, is expelled and oxygen is drawn in.

This lack of chemical intuition can be likened to 'sound blindness'. Sound blindness was the phrase coined by Franz Boas in the late 19th century (Boas 1889:47-54) to describe the incapacity of American anthropologists to discern the subtle difference in tone which gave rise to new meanings in languages they encountered in the islands of the South Pacific. These languages, much like Mandarin, are tonal languages. The anthropologists, used to European languages, simply could not hear the tonal variation. In a similar manner, most people are chemically blind. This gives rise to nonsensical claims such as beauty products being 'chemical free' (Shivaram 2019:12). 'Chemical' is just another word for a 'distinct compound or substance' (OED Online 2021), so any particular substance is a chemical, and is made of matter.

From a chemist's point of view, the building blocks of matter are atoms. The types of atoms that make up a particular bit of matter, and the way those atoms are arranged, affect the properties and the behaviour of that matter. Changes in the way atoms are arranged can affect the melting point of a substance, how quickly it reacts and even its colour. Changes in the world we see around us macroscopic changes - have their origin in the sub-microscopic realm of atoms and molecules.

Note: Special Collection: Challenging Building Blocks, sub-edited by Chris Jones (Stellenbosch University) and Juri van den Heever (Stellenbosch University). 
An element is a substance that only consists of one type of atom. (The general chemistry discussed in this article can be found in any good introductory undergraduate chemistry textbook, e.g. Brown et al. 2013. We have thus not referenced every one of these statements that are general chemical knowledge, and refer the interested reader to such a text for more detail). For example, oxygen only contains oxygen atoms, and sulfur contains only sulfur atoms. Elements cannot be decomposed into simpler substances, so an element is a substance that cannot be purified any further. A compound is a substance that contains more than one type of atom: water is a compound made up of both oxygen and hydrogen atoms. Under the right conditions, water can be decomposed into oxygen and hydrogen. Atoms can be combined in a variety of ways to give the vast array of substances we see around us.

Physics, chemistry and biology are deeply interrelated, but occupy different levels of complexity. Particle physics is concerned with fundamental particles - quarks, bosons, muons and the like. Quarks combine in various ways to give the sub-atomic particles protons and neutrons. Biological systems are made up of complex aggregations of enclosed environments with highly controlled chemical reactions. Self-replication of the aggregate, which could be a cell, an organism or even a piece of nucleic acid which has found a way to program cells of another organism to replicate it (a virus!), is a key feature of biological systems. Chemistry falls between these two, and tries to build a bridge between them (Lehn 2014).

\section{How did chemistry begin?}

In ancient times, the craft of alchemy began with the interrogation of the ways in which matter could be changed. Alchemical activity is observed in many ancient civilisations. The very characterisation of the bronze age and iron age indicates that metallurgy was in operation (Amzallag 2009:497-519). There is evidence of use of the technology of metallurgy in the artefacts found through archaeology across the world. In some civilisations, such as ancient Egypt or ancient Greece, we also have written records of a diversity of exploration. In ancient times, the elements were conceived as a combination of four or five factors. In ancient Greece, these were water, earth, fire and air; in China, they were wood, fire, earth, metal and water.

Alchemy is strongly associated with the desire to change base metals to gold. We now know that this was a fool's errand. However, alchemy is also the forerunner of modern pharmacology (Read 1995). Making tea from willow bark to treat a headache or other pain, we now recognise as the extraction of acetylsalicylic acid. Acetylsalicylic acid is the chemical name for the compound which is sold under the brand name of Aspirin.

Chemistry as a science emerged as the technology of measurement became accurate. The capacity to weigh matter accurately was a critical turning point (Fabbrizzi 2008:1501), along with the Enlightenment drive to understand and explain what was being observed. It became clear through measuring the mass of the materials before and after a chemical transformation had taken place that the total mass of the substances before the reaction and after the reaction is the same (Read 1995). This is called the law of conservation of mass (see Dalton's atomic theory below). The old 'elements' of water, earth, fire and air were no longer sufficient to explain the observations scientists were making, and a new theory was needed.

\section{Development of atomic theory}

The idea of atoms, that is, the idea that matter is composed of discrete indivisible units, has been around for a very long time. Centuries BC, the Indian and Greek philosophers argued for the existence of a fundamental building block of matter. The very word 'atom' comes from the Greek átomos, which means uncuttable (OED Online 2021).

The modern idea of an atom really started with John Dalton, who was a schoolteacher in Manchester in England in the early 1800s (Gribbin 2003). Dalton used the idea of atoms as indivisible units to explain the law of conservation of mass. As we saw above, this law states that the mass of substances formed by a reaction is the same as the mass of substances going into the reaction. Because matter cannot be created or destroyed, the mass of the products of a reaction must equal the mass of the reagents. In Dalton's description of atoms, all the atoms of a particular element (see above) are the same as each other, and they have a particular size and mass. Each type of element has its own type of atom, and in a chemical reaction, these atoms change the way they are connected to one another. In other words, the number and type of atoms do not change in a chemical reaction. All that changes is the way the atoms are arranged. Because each type of atom has its own defined mass, the mass of all the atoms before a reaction is the same as the mass of those same atoms after the reaction (the rearrangement) has taken place.

Dalton also used atoms to explain another law known at the time, the law of constant composition, which states that all samples of a compound have the same composition, that is, the same proportions by mass of the constituent elements. Any sample of water, when decomposed, will always yield the same relative masses of hydrogen and oxygen. Dalton said that there are as many different types of atoms as there are elements, and that atoms of different elements have different masses. As mentioned above, he explained chemical reactions as changes in the way atoms are grouped together. This is still how chemical reactions are understood today (Figure 1).

The simple model of an atom as a hard sphere has been greatly advanced over the past century, particularly as a result of the understanding gained from quantum mechanics. We now know that atoms are not indivisible at all, in contrast to what the Greeks believed. Atoms contain protons, neutrons 


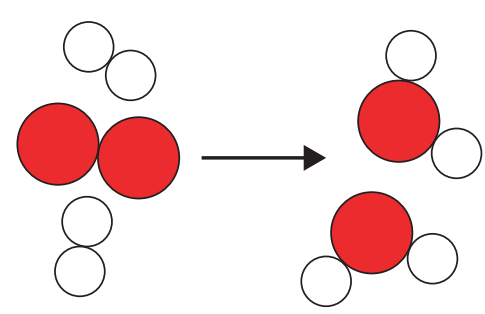

FIGURE 1: A scheme of a simple reaction, where spheres represent atoms Different types of atoms are indicated by different colours and sizes. The reaction involves rearrangement of the atoms, but there is same number of each type of atom both before and after the reaction. This particular scheme is a representation of the reaction of hydrogen and oxygen to give water, which a chemist would write as $2 \mathrm{H}_{2}+\mathrm{O}_{2} \rightarrow 2 \mathrm{H}_{2} \mathrm{O}$ (vide infra).

and electrons. The charge on an electron is $-1.602 \times 10^{-19}$ coulombs (C), and that of a proton is $1.602 \times 10^{-19} \mathrm{C}$. Usually (so we do not have to keep writing $10^{-19}$ !), we express these charges as multiples of the fundamental charge, so usually we would say the charge on the electron is -1 , and on the proton, it is +1 . Protons and neutrons have mass, which again are very small, and so we usually express them in unified atomic mass units $(\mathrm{u})$, where $1 \mathrm{u}=1.66054 \times 10^{-24} \mathrm{~g}$. Protons and neutrons both have a mass close to $1 \mathrm{u}$. The electron has a far smaller mass of $5.486 \times 10^{-4} \mathrm{u}$. By far the majority of the mass of an atom comes from protons and neutrons.

Atoms are very small - between $1-5 \times 10^{-10} \mathrm{~m}$. The protons and neutrons in an atom are bound together in the nucleus by the strong nuclear force, one of the four fundamental forces of nature. The atomic nucleus is extremely small, and the vast majority of the space in an atom is where the electrons reside. If the atoms were of the size of a cathedral, the nucleus would be about the size of a fly (Cathcart 2005).

How do we know about all these extremely tiny particles? Their existence was discovered by a series of scientists who carried out some careful and clever experiments in the late 19th and early 20th centuries. In 1897, Thompson discovered the electron, and established that this particle, which he found in cathode rays, is significantly smaller than the atom (Thompson 1897:293-316). This was a significant finding: the atom is not indivisible, as was thought, but rather is made up of smaller subatomic particles. Another deeply important idea from Thompson's (1897) work, that subatomic particles are the same in all atoms, is best expressed in his words:

$[\ldots A] l l$ matter - that is, matter derived from different sources such as hydrogen, oxygen \& c. - is of one and the same kind; this matter being the substance from which all the chemical elements are built up. (pp. 293-316)

The next big step in our understanding of atomic structure was taken by Ernest Rutherford, under whose guidance the well-known 'gold foil' experiment was carried out. In fact, this was a series of experiments carried out by Ernest Marsden and Hans Geiger (who also developed the Geiger counter) between 1908 and 1913, while working under Rutherford. Geiger and Marsden fired a stream of alpha particles, which are emitted by some radioactive substances, at various substances, including thin sheets of gold foil. They observed that some of the alpha particles went straight through the sheet, but others were deflected and some bounced directly back. Based on the understanding of the atom at the time, this was a very unexpected result. In Rutherford's own words:

It was quite the most incredible event that has ever happened to me in my life. It was almost as incredible as if you fired a 15-inch shell at a piece of tissue paper and it came back and hit you. (Andrade 1968:247-250)

Rutherford explained these results by suggesting that most of the mass of an atom is concentrated in a small, positive central core, which we now know as the nucleus. Only those alpha particles which came into contact with the nucleus would be bounced back.

The final constituent of atoms, the neutron, was discovered in 1932 by James Chadwick. Interestingly, Thompson and Rutherford, as well as Rutherford and Chadwick, worked together at different stages of their careers (Gribbins 2003).

In 1913, Niels Bohr introduced the idea of quantisation to atomic theory (Bohr 1913:1-25). Quantisation comes from the Latin word quanta, meaning 'how much', and refers to the fact that some things only come in particular values. For example, money is quantised: we have a R1 coin, a R2 coin and a R5 coin, but there is no R3 coin - coins are quantised and only have particular values. In the same way, Bohr proposed, the energy of an electron in an atom is quantised: it can only have particular values. In the Bohr model of the atom, electrons revolve around the nucleus, like planets revolve around the sun, in orbits of fixed radii. The energy of the electron depends on the radius of its orbit.

This 'solar system' model is the way atoms are commonly depicted, but it is almost entirely wrong. Electrons have properties of both particles and waves (they can interfere or be diffracted like waves, but can be detected at a point like particles). This is known as 'wave-particle duality' and is a fundamental aspect of how all matter behaves. We do not normally notice the wave nature of larger particles because they have extremely short wavelengths. Quantum mechanics and the wave theory of the electron were developed in the mid-1920s by Heisenberg, de Broglie and Schrödinger, each of whom made profound contributions to our understanding of the behaviour of electrons (Challoner 2018). Part of what quantum mechanics tells us is that we cannot specify the exact position of an electron, we can only talk about the probability of finding an electron in a particular region of space. Chemists now describe electrons as residing in orbitals, which are mathematical functions with wave character that tell us something about where the probability of finding a particular electron is the greatest.

The ideas of quantum mechanics also have some profound philosophical implications. For example, if, as specified by 
quantum mechanics, we cannot describe the precise position or momentum of a particle, we cannot use Newton's Laws to describe where the particle will go next. In other words, we cannot predict the future by precisely describing the position and momentum of everything in the Universe. The interpretation and meaning of quantum theory, and exactly what it tells us about reality, is still an open conversation.

\section{The atom and the periodic table}

As we have discussed above, an atom is made up of protons, neutrons and electrons. The thing that makes one type of atom different from another type of atom is the number of protons in its nucleus. For example, if an atom has 6 protons in its nucleus, it is an atom of carbon. An atom of oxygen has 8 protons in its nucleus. The number of protons in the nucleus is called the atomic number of the element. Each element has its own atomic number, and these numbers are always whole numbers.

Atoms are electrically neutral, so there must be the same number of electrons as protons in an atom. Atoms can, however, differ in the number of neutrons they have in the nucleus. Usually, it is close to the number of protons, but it can be more or less. For example, carbon has 6 protons and usually has 6 neutrons, but it can also have 7 or 8 neutrons. Atoms with identical numbers of protons but different numbers of neutrons are called isotopes. The mass number is the sum of the protons and neutrons (because they each essentially have a mass of 1). Isotopes have the same atomic number but different mass numbers. Carbon with 8 neutrons is known as carbon-14 (because it has a mass number of 14: 6 protons +8 neutrons $=14)$, and it is this isotope of carbon that is used in carbon dating, which is used to date samples that came from living tissue. Living things have the same amount of carbon-14 in them as the environment until they die. After they die, they no longer get any more of this isotope into their system from the environment. Carbon-14 is radioactive, and so it decays over time. By measuring the amount of carbon-14 remaining in a sample, we can tell how long ago it died.

The underlying structure of the atoms that make up the elements is reflected in the periodic table, which lists all the known elements. The elements in each column of the periodic table have the same arrangement of their outermost electrons. Amazingly, the periodic table was developed before the electron was even discovered - Mendeleev's periodic table, which is essentially the one we use now, was published around 1871, and the electron was only discovered in 1896 (Strathern 2000).

So how was the periodic table put together? Scientists had noticed that there are relationships in the properties and chemistry of the elements. In fact, if the elements are arranged in order of increasing atomic mass, there are periodic variations in the properties of the elements. For example, there is a relationship between positions of elements and stoichiometry of simple compounds (stoichiometry refers to how many of each type of atoms are in a compound). The metals sodium $(\mathrm{Na})$ and potassium $(\mathrm{K})$, which can be found in the left-hand column of the periodic table, both form compounds with a 1:1 ratio with chlorine, whereas magnesium $(\mathrm{Mg})$ and calcium $(\mathrm{Ca})$, from the second column, form compounds with a 1:2 ratio with chlorine. Based on these observations, elements with similar properties were grouped together, leading ultimately to the periodic table as we know it today.

The periodic table is a visual representation of the periodic law, which states that the elements show approximate repetition in their properties at regular intervals. So, lithium and sodium, separated by 8 , have similar properties. Fluorine and chlorine are also separated by 8 , and also have similar properties. The properties of the elements are periodic hence the periodic table.

Mendeleev made the most important contributions to the development of the periodic table. In fact, he noticed that some elements did not fit the position implied by their atomic mass, so he left gaps and moved things around so that the periodicity in properties was maintained. It was later shown that the order was based on atomic number and electronic structure - we now have the theoretical understanding of the experimentally established periodic law. Mendeleev actually made predictions about missing elements and their properties. For example, he predicted the existence of an element which he called eka-silicon, giving predictions of its melting point, boiling point and a number of other important properties. We now know the element as germanium (Ge), and Mendeleev's predictions of its properties were all remarkably close to what has been measured (Greenwood \& Earnshaw 1984).

There are currently 118 known elements in the periodic table: it looks like it is full. The first 94 elements occur naturally; the remaining 24, americium to oganesson (95-118), occur only when synthesised in laboratories. These synthetic elements are made in very small quantities by bombarding atoms of one element with another, or with other particles, in nuclear reactors, particle accelerators or atom bomb explosions. No element heavier than einsteinium (element 99) has ever been observed in macroscopic quantities in its pure form, nor has astatine (element 85). But, are there more elements waiting to be discovered? This is still a hotly debated topic, and several research groups around the world are actively engaged in trying to make previously undiscovered elements. A theoretician, Pekka Pyykkö, has published a periodic table where he has calculated the positions of the elements up to atomic number 172 (Pyykkö 2011:161-168). These elements, if they are ever made, will probably be very unstable and are not likely to exist for very long.

The periodic table is of great use to chemists because of its predictive power. As mentioned above, Mendeleev used the periodic table to predict the existence of unknown elements. Chemists now use the periodic table to predict the properties an element will have, and how it will bond to other elements. 
For example, the periodic table allows us to predict which combinations of elements are most likely to form semiconductors, and which compounds will lead to acid rain.

\section{Symbols in chemistry}

There is little real understanding of chemistry without grappling with the periodic table. But the symbolic density in the periodic table is substantial: the use of a symbol for an element ( $\mathrm{C}$ for carbon, $\mathrm{Cu}$ for copper), the significance of the physical position of the element in the table and so on. This is all before any kind of association of elements into molecules and compounds is taken into consideration. Water, symbolised as $\mathrm{H}_{2} \mathrm{O}$, is a combination of two hydrogen atoms with a single oxygen atom (hence, the subscript 2 after hydrogen). But the properties of water are distinct from hydrogen gas and oxygen gas.

The use of symbols in chemistry is by no means new. Symbols were used extensively by alchemists. With the development of alchemy came an element of protecting intellectual property. The power of transformation of matter took on an esoteric flavour and with this 'recipes' began to be coded in symbols:

Alchemists were essentially practitioners, mainly concerned with producing new substances or devising new procedures for preparing precious materials at low cost. They were not interested in or capable of establishing theories for the rationalization and explanation of the many experiments described. (Fabbrizzi 2008:1501)

Ancient alchemists are similar then, to many modern chefs. The all-important product is the object of desire, rather than understanding the science of the process which is occurring. It was only in the 18th century with the development of the precision balance (Fabbrizzi 2008:1501) that the mystery of transmutation inherent in alchemy gave way to the decidedly more grounded, and infinitely more powerful science of chemistry.

While the alchemists' agenda of using symbols may have been driven by the desire to retain the control of the knowledge, modern chemistry is inseparable from the use of complex symbols. Each branch of chemistry has its own subset of symbols which have been developed to convey information. The representation of the chemical equation will be discussed in some detail here because anyone who has studied science at high school is likely to have encountered these equations. It is far easier to show the power of the chemical equation using a simple example. Here, we have chosen a reaction which many will have encountered effervescent tablets. These are usually large tablets which one is required to dissolve in water before drinking. They 'fizz' as carbon dioxide is released as they dissolve. There are many examples of effervescent tablets as a mode of drug delivery. Vitamins, minerals, headache tablets and antacids are the most common. Essentially, these are all based on the same chemical reaction (Chen \& Yaung 2002:848). To the chemist, the general reaction would be written as:
$\mathrm{HA}+\mathrm{NaHCO}_{3} \rightarrow \mathrm{NaA}+\mathrm{CO}_{2}+\mathrm{H}_{2} \mathrm{O}$

There is a great deal encoded in this equation. With the exception of ' $\mathrm{A}$ ', all the symbols can be found in the periodic table. ' $A$ ' here is used to symbolise any acid - this could be ascorbic acid (vitamin C), acetylsalicylic acid (aspirin) etc., and will be particular to the medicine. The rest of the substances are common to most of these kinds of tablets. Thus, a slightly less 'encoded' version could be written as:

An acid + sodium bicarbonate react to form a sodium salt of the acid + carbon dioxide + water.

While it is certainly easier to read, this simplified version slips into an awkward no man's land of being neither a chemical equation, nor being completely accessible to the lay reader. As chemists, we lose the clarity of communication on the conservation of matter: if you count the atoms of each type before the arrow, they will equal the atoms of each type after the arrow, but they may not be coupled to the same thing. For example, there are two $\mathrm{H}$ atoms before the arrow; one in $\mathrm{HA}$, the acid, and one in $\mathrm{NaHCO}_{3}$, sodium bicarbonate. After the arrow, both $\mathrm{H}$ atoms are present in a single water molecule. Importantly, this detail in the chemical equation also means that we can know exactly how much of each substance we will need to ensure that we do transform all the acid into the salt of the acid. One acid molecule reacts with one molecule of sodium bicarbonate. Correct balancing of the equation (making sure that both sides have the same number of each type of atom) is the bane of many high school learners' life, and is known as reaction stoichiometry.

As we have already seen, chemical combination matters. The properties of water are not obviously related to hydrogen or oxygen. From a pharmaceutical point of view, the acid, HA may not be particularly soluble in water, and therefore may not be in the best form for absorption through the wall of the intestine. Changing the acid into its salt, $\mathrm{NaA}$, may well make it more water soluble and therefore make it more available for absorption. So, the pharmaceutical manufacturer makes use of a chemical reaction, which you perform in a glass when you add the effervescent tablet to the water, to deliver the ascorbic acid (vitamin C) to you in a form you can more easily absorb (sodium ascorbate). And the reaction comes with the delightful sensory bonus of fizzing as carbon dioxide is released.

All around us, on a daily basis, we employ chemical reactions. The petrol in your car is reacting with oxygen to form carbon dioxide and water, alongside releasing a substantial amount of energy. That reaction can be written in the condensed form of the chemical equation, and calculations on how much energy is released and how much oxygen is required will impact the design of the engine. The act of cooking always involves chemical reactions. The study of changing the properties of matter through the application of heat surely dates back to the harnessing of fire for cooking. The definition of meat is 'muscle tissue that 
has undergone physical and chemical change' (Provost et al. 2016). The cooking of meat entails several processes. Collagen, one of the macromolecules present in the meat, will shrink upon heating. Collagen is a protein - a complex structure comprising a long chain of amino acids bonded together, held in a particular three-dimensional shape by weaker intermolecular forces. The 'shrinkage' of the collagen is a result of the disruption of these relatively weak intermolecular forces. The three-dimensional structure is no longer held together and so the collagen no longer holds the structure of meat together so strongly. The result is that meat is more 'tender' (Provost et al. 2016). There are clearly other changes to the meat, such as browning etc., which are also chemical changes.

Any chemical reaction will involve the breaking and making of chemical bonds. What constitutes a chemical bond? How do atoms combine to form compounds?

\section{Chemical bonding}

As we have mentioned in passing, atoms can combine to form molecules, which contain two or more atoms joined together. A molecule can be made up of atoms of the same type - for example, the oxygen molecule, $\mathrm{O}_{2}$. Molecules can also be made up of different kinds of atoms, for example, water is $\mathrm{H}_{2} \mathrm{O}$. Note that the number of each atom type in a molecule is very important $-\mathrm{H}_{2} \mathrm{O}$ (water) is a very different substance from $\mathrm{H}_{2} \mathrm{O}_{2}$ (hydrogen peroxide)!

How do atoms combine with one another in order to form molecules? What holds the building blocks of matter together? We now know that it is the interaction between the electrons in atoms that holds the atoms together in a molecule. This can happen in several ways, each of which results in a class of substances with distinct properties (Figure 2).

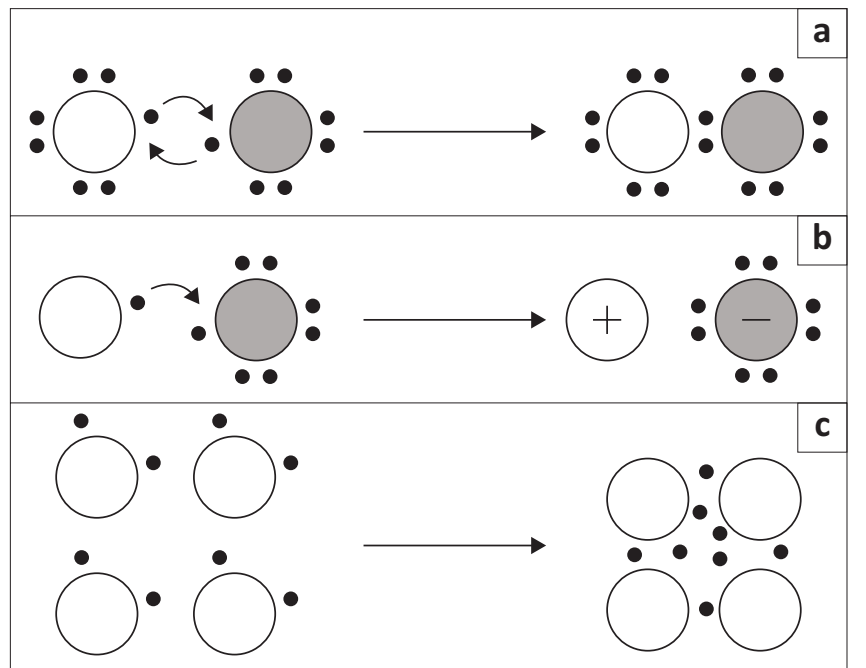

FIGURE 2: Types of bonding. (a) Covalent bonding, in which atoms are held together by a shared pair of electrons. (b) lonic bonding, where transfer of an electron from one atom to another results in a cation (positively charged) and an anion (negatively charged), which are held together by electrostatic forces. (c) Metallic bonding, where electrons are shared across many atoms in a 'sea'.

\section{Covalent bonding (Figure 2a)}

In a covalent bond, electrons are shared between atoms, and the shared electrons are what hold the atoms together. Atoms will share electrons with each other so that each atom has a stable number of electrons around it (usually eight). In order to share electrons, the atoms must be close to each other, and the electrons must stay between the two atoms. This is called a covalent bond, and it holds atoms together quite strongly. A large amount of energy needs to be added to a molecule to break apart the atoms held together by a covalent bond.

\section{Ionic bonding (Figure 2b)}

Sometimes, instead of electrons being shared between two atoms, an electron is completely transferred from one atom to another. When this happens, the atom that gains an electron has a negative charge, because it has more electrons than protons. This is called an anion. The atom that lost an electron has a positive charge, and is called a cation. Because anions have a negative charge, and cations have a positive charge, they are attracted to one another through an electrostatic force. This electrostatic interaction between a cation and an anion is what holds the atoms together. This is called ionic bonding.

\section{Metallic bonding (Figure 2c)}

In metallic bonding, all the electrons in the substance are shared over all the atoms. A simple image is that of a 'sea' of electrons. Because the electrons are not strongly associated with any one atom, they can move through the solid, which is why metals can conduct electricity.

An important point here is that the nature of the bonding, that is, the nature of the way electrons are shared between atoms, is what gives the resulting material its properties. By understanding the bonding, we can control and develop the properties of materials. For example, understanding that a 'sea' of electrons leads to conductivity has allowed researchers to design polymers (very large molecules that are usually covalently bonded), where some of the electrons are less tightly bound to a single atom. These materials, when doped (extra electrons are added or removed), conduct electricity (Nobel Prize organisation n.d.).

In recent years, the existence of a new type of bonding, called charge-shift bonding, has been proposed (for a perspective, see Shaik et al. 2009:443-449). Our scientific understanding of bonding, and how the building blocks of matter are held together, is still growing and developing.

\section{States of matter}

We have discussed how chemistry is the study of chemical reactions as illustrated through the effervescent tablet. But chemistry is also the study of changing states of matter. Matter can exist in various forms. The substance that we change from one form to another on a daily basis is water. Ice is the solid form of water, which we can change to the liquid 
form by melting it, and then to the gaseous form by applying heat until it boils.

There is a classic in the education literature which asks incoming postgraduate students in chemistry what is in the bubbles that form when water boils (Gabel, Samuel \& Hunn 1987:695). The correct answer is water molecules in the gaseous state. Alas, far too many students answered a mix of hydrogen gas and oxygen gas! (Hydrogen and oxygen react explosively to form water!) When a molecular substance, which has covalent bonds between a small number of atoms, changes state, the bonds between the atoms of the molecule do not change. The $\mathrm{H}_{2} \mathrm{O}$ molecule remains intact when water boils, but the intermolecular forces (the weaker bonds between the molecules) are disrupted. This is why it does not take too much energy to boil water - it boils at $100{ }^{\circ} \mathrm{C}$. However, if you want to boil table salt, much more energy is required. This is because salt $(\mathrm{NaCl})$ is made up of sodium cations and chloride anions bound together by ionic bonds. To boil salt, you would need enough energy to break all of the ionic bonds, resulting in cations and anions in the gas phase. $\mathrm{NaCl}$ boils at $1465^{\circ} \mathrm{C}$.

An age-old technology which takes advantage of changes in the state of matter is distillation. This process, most commonly used to gain a more concentrated solution of alcohol, is a separation technique. Alcohol, which is mostly ethanol, has a slightly lower boiling point than water. Beer and wine can be produced through simple fermentation: yeast is used to convert sugar from the grain or grapes into alcohol. To get spirits, one needs to distil. The distillation apparatus allows one to boil the liquid and to cool the vapour into a second container. Because ethanol boils at a lower temperature, it enters the gas phase first, and can be cooled and collected. Ethanol and water cannot be completely separated this way, but the percentage of alcohol in the mixture can still be increased quite substantially.

\section{So what?}

For a person who has been 'chemically blind', the implications of a chemical understanding of matter may still not be clear. For a moment, simply pause and look around you. The clothes that you are wearing, the beverage on your desk, any paper or printed item, the computer, the glass of the window, the varnish on your desk: the production of every single one of those items involves the utilisation of chemistry. So many of the things we take for granted as being an essential part of life involve chemical processes. And the more thoroughly we understand the science, the better the technology we can build. You may not have a chemical understanding of the world, but the world you inhabit is utterly infused by chemistry and has been profoundly shaped in and through the advancement of the science. The world as you know it would not be as it is were it not for chemistry.

In this time of the COVID-19 pandemic, two things have become ubiquitous: face masks and hand sanitiser. The face mask provides physical protection. It will trap small water droplets containing the virus. Hence, one needs to wash them frequently, and their efficacy is entirely dependent on the pore size of the material. More layers, with a material of small pore sizes, will provide a more efficient barrier. Hand sanitiser (or soap and water) operates on a chemical level. The virus is encapsulated in a membrane. The alcohol in the sanitiser disrupts the membrane. Without the membrane, the virus no longer has the biological docking equipment to hijack human cells, and without the human cell, the virus cannot replicate. While soap does the same thing, the additional action of the flow of water means that any virus that is present gets washed away.

You may not need to know the chemistry to use hand sanitiser or a mask. But if you understand the chemistry, it will surely assist in understanding where you have to make choices about what can be substituted.

Deep understanding of the way the building blocks of matter come together to give the things we see around us ultimately allows us to manipulate matter, giving us control. This is most powerfully demonstrated in two examples: the development of the atomic bomb, and the development of chemotherapeutics, drugs and vaccines. Our ability to manipulate the building blocks of matter comes with some responsibility. This is summarised beautifully in the words of Linus Pauling, a hugely influential chemist, and the only person to win two unshared Nobel prizes, one for chemistry and one for peace:

Every aspect of the world today - even politics and international relations - is affected by chemistry.

and, importantly:

The two factors that will determine the nature of the world of the future are the knowledge that we possess and the decisions that we make about how to use that knowledge. (Pauling 1984:54-56)

\section{Acknowledgements Competing interests}

The authors declare that they have no financial or personal relationships that may have inappropriately influenced them in writing this article.

\section{Authors' contributions}

Both authors contributed equally to the preparation of this manuscript.

\section{Ethical considerations}

This article followed all ethical standards for research without direct contact with human or animal subjects.

\section{Funding information}

The publication of this article was supported financially by Stellenbosch University. 


\section{Data availability}

Data sharing is not applicable to this article as no new data were created or analysed in this study.

\section{Disclaimer}

The views and opinions expressed in this article are those of the authors and do not necessarily reflect the official policy or position of any affiliated agency of the authors.

\section{References}

Amzallag, N., 2009, 'From metallurgy to bronze age civilizations: The synthetic theory' American Journal of Archaeology 113(4), 497-519. https://doi.org/10.3764/ aja.113.4.497

Andrade, E.N.D.C., 1968, 'Some reminiscences of Ernest Marsden's days with Rutherford at Manchester', Notes and Records of the Royal Society of London 23(2), 247-250. https://doi.org/10.1098/rsnr.1968.0025

Blackie, M.A., 2014, 'Creating semantic waves: Using Legitimation code theory as a tool to aid the teaching of chemistry', Chemistry Education Research and Practice 15(4), 462-469. https://doi.org/10.1039/C4RP00147H

Boas, F., 1889, 'On alternating sounds', American Anthropologist A2(1), 47-54. https://doi.org/10.1525/aa.1889.2.1.02a00040

Bohr, N., 1913, 'I. On the constitution of atoms and molecules', The London, Edinburgh, and Dublin Philosophical Magazine and Journal of Science 26(151), 1-25. https:// doi.org/10.1080/14786441308634955

Brown, T.L., LeMay, H.E., Bursten, B.E., Murphy, C., Woodward, P., Langford, S. et al., 2013, Chemistry: The Central Science, 3rd edn., Pearson Australia, Camberwell.

Cathcart, B., 2005, The fly in the Cathedral: How a group of Cambridge scientists won the International race to split the atom, Farrar, Straus and Giroux, New York, NY.

Challoner, J., 2018, The Atom: The building block of everything, Ivy Press, London.

Chen, Y.-H. \& Yaung, J.-F., 2002, 'Alka-Seltzer Fizzing-determination of percent by mass of NaHCO in Alka-Seltzer tablets: An undergraduate general chemistry experiment', Journal of Chemical Education 79(7), 848. https://doi.org/10.1021/ed079p848
Fabbrizzi, L., 2008, 'Communicating about matter with symbols: Evolving from Alchemy to chemistry', Journal of Chemical Education 85(11), 1501. https://doi. org/10.1021/ed085p1501

Gabel, D.L., Samuel, K. \& Hunn, D., 1987, 'Understanding the particulate nature of matter', Journal of Chemical Education 64(8), 695. https://doi.org/10.1021/ ed064p695

Greenwood, N.N. \& Earnshaw, A., 1984, Chemistry of the elements, Pergamon Press Ltd., Oxford.

Gribbin, J., 2003, Science: A history, Penguin Books Limited, London.

John, A.J., 2021, 'The building blocks of the universe', HTS Teologiese Studies/ Theological Studies 77(3), a6831. https://doi.org/10.4102/hts.v77i3.6561

Lehn, J.-M., 2014, Perspectives in chemistry (1st Part), YouTube, viewed 28 April 2021, from https://www.youtube.com/watch?v=9RDYaeQvd9w.

Nobel Prize Organisation, n.d., The Nobel Prize in chemistry 2000, viewed 02 May 2021, from https://www.nobelprize.org/prizes/chemistry/2000/summary/.

OED Online, 2021, Oxford University Press, March 2021, viewed 29 April 2021, from www.oed.com/view/Entry/115083.

Pauling, L., 1984, 'Priestley medal address: Chemistry and the world of tomorrow', Chemical and Engineering News 62(16), 54-56. https://doi.org/10.1021/cenv062n016.p054

Provost, J.J., Colabroy, K.L., Kelly, B.S. \& Wallert, M.A., 2016, The science of cooking: Understanding the biology and chemistry behind food and cooking, John Wiley \& Sons, Hoboken, NJ.

Pyykkö, P., 2011, 'A suggested periodic table up to $Z \leq 172$, based on Dirac-Fock calculations on atoms and ions', Physical Chemistry Chemical Physics 13(1), 161-168. https://doi.org/10.1039/COCP01575J

Read, J., 1995, From alchemy to chemistry, Courier Corporation, New York, NY.

Shaik, S., Danovich, D., Wu, W. \& Hiberty, P.C., 2009, 'Charge-shift bonding and its manifestations in chemistry', Nature Chemistry 1(6), 443-449. https://doi org/10.1038/nchem.327

Shivaram, K., 2019, 'Disproportionate chemical exposure: How the marketing and production of beauty products in the United States impose health burdens on women', Intersect: The Stanford Journal of Science, Technology, and Society 12(3), $1-11$.

Strathern, P., 2000, Mendeleev's dream: The quest for the element, St Martin's Press, New York, NY.

Thompson, J.J., 1897, 'XL: Cathode rays', The London, Edinburgh, and Dublin Philosophical Magazine and Journal of Science 44(269), 293-316. https://doi. org/10.1080/14786449708621070 